\title{
UNDERSTANDING THE INTERACTION BETWEEN VIRTUAL DESIGN, CONSTRUCTION AND LEAN CONSTRUCTION
}

\author{
Maria Guadalupe Mandujano Rodriguez ${ }^{1}$, Luis Fernando Alarcon Cardenas ${ }^{2}$, \\ Bhargav A. Dave ${ }^{3}$, Claudio Mourgues ${ }^{4}$, and Lauri Koskela ${ }^{5}$
}

\begin{abstract}
There have been important advances regarding the synergies between Building Information Modeling (BIM) - as part of Virtual Design and Construction (VDC) - and Lean Construction. However, the literature does not fully explore the nature of these synergies nor the conceptual reasons behind them. This better understanding of these synergies would allow the Architecture, Engineering and Construction (AEC) industry to achieve better Lean and VDC implementations and would provide a stepping-stone for the academia to continue building on these synergies. This article presents a thorough literature review based on leading international journals, conference proceedings and books, to explore the synergies between Lean Construction and VDC, including BIM (product), process and organization modeling. As part of this review, the article tests mechanisms about interaction mechanisms, previously mentioned in the literature. The findings indicate that using the entire VDC framework, the positive interactions between Lean and VDC increased significantly with respect to the same analysis restricted to the interaction between Lean and BIM. Identifying these new interactions and interaction mechanisms can help the AEC industry take a more holistic approach and generate improvements in every project phase.
\end{abstract}

\section{KEYWORDS}

Lean construction, collaboration, BIM, VDC, synergy.

CEO, MARO Consulting, Huejutla de Reyes, Hidalgo, Mexico, Phone +52-789-89-609-69, info@maroconsulting.mx, orcid.org/0000-0002-3588-2002

2 Professor of Civil Engineering, Dept. of Construction Engineering and Management, Pontifica Universidad Católica de Chile, Escuela de Ingeniería, Casilla 360, Correo 22, Santiago de Chile, Phone (56 2) 2354-4201, lalarcon@ing.puc.cl, orcid.org/0000-0002-9277-2272

3 Senior Researcher, Dept. of Computing, Aalto Univ., Espoo 02150, Finland, Phone +358-50-4364717, bhargav.dave@aalto.fi, orcid.org/0000-0003-4228-506X

4 Assistant Professor of Civil Engineering, Dept. of Construction Engineering and Management, Pontificia Universidad Católica de Chile, Escuela de Ingeniería, Casilla 360, Correo 22, Santiago de Chile, Phone (56 2) 2354-4245, cmourgue @ing.puc.cl, orcid.org/0000-0001-6706-2255

5 Professor, School of Art, Design, and Architecture, Univ. of Huddersfield, Huddersfield HD1 3DH, UK, Phone: +44-01484-472892, 1.koskela@ @ud.ac.uk, orcid.org/0000-0003-4449-2281 


\section{INTRODUCTION}

The major challenges facing the architecture, engineering, and construction (AEC) industry have created a new way of working, forcing companies to use new methodologies such as virtual design and construction (VDC) (Kunz and Fischer 2020). VDC is perceived as an approach that will help the AEC industry achieve better results by increasing the value of projects, reducing their costs, improving productivity, and creating other positive results (Lee et al. 2020). In a similar way, the Lean Construction philosophy can be used as a conceptual framework for VDC implementation because the impacts of VDC can be directly associated with Lean Construction principles (Alarcon et al. 2013). In view of this development, there is a growing need to make VDC users aware of Lean Construction principles, as well as a need to make Lean Construction users aware of the benefits of VDC (Mandujano 2019).

There have been important advances regarding the synergies between building information modeling (BIM), as part of virtual design and construction (VDC) and Lean Construction (Kunz and Fischer 2020; Mandujano 2017; Mandujano et al. 2016). Despite these advances, previous studies have been focused primarily on product modeling and Lean Construction synergies, leaving aside the process and organizational components. It is therefore important to understand a) the full extent of the synergies between VDC, including BIM (product), process and organization modeling, and Lean Construction; b) the nature of these synergies (i.e., how strong or weak, direct or indirect, etc.); and c) the reasons and conceptual explanations of why these synergies exist.

The remainder of this paper is structured as follows. Section 1 explains the difference between VDC and BIM. Section 2 reports the state of the art of VDC and Lean Construction. Section 3 presents the study's research method. Section 4 develops the interactions matrix. These new interactions complete the matrix proposed by Sacks et al. (2010) but also helps analyze the current VDC implementation from a Lean perspective and can help identify new VDC and Lean adoption strategies. Section 5 tests the four mechanisms proposed by Dave et al. (2013). Section 6 identifies the key gaps in the literature. Finally, in section 7 the conclusions and the implications for further research are outlined.

\section{BACKGROUND}

The literature is ambiguous about the differences between VDC and BIM. As a result, some companies have sold BIM as simply a software platform, setting aside the core of the methodology: collaborative work (Mandujano 2017). In this paper, we continue define VDC as mentioned by Kunz and Fischer (2011): "The use of integrated multidisciplinary performance models of design-construction projects to support explicit and public business objectives". Building Information Models (BIM) represent the form/scope of the product, which is a crucial but a partial representation of both the total perspective of a project and the information about a project represented in the VDC framework and a POP model (Alarcon et al. 2013). In this paper, we continue to define BIM as mentioned by Eastman et al. (2018): "A digital database of a particular building that contains information about its objects".

At the outset, it seems that the definition of BIM is slightly narrower and focuses on the production of a 3D virtual model that represents the physical reality, hence excluding the process element. On the other hand, VDC seems to focus on the overarching process, and takes BIM (or 3D modeling) as one of the tools and goes on to include 4D production, 
organizational and process modeling tools, and collaborative techniques as part of the approach. However, the authors would rather like to argue that this broader or inclusive definition is a somewhat modern development and the origins of the concept have deeper technological underpinnings. The authors would like to suggest that while the academia and industry have now realized the value of including people (organizational) and process aspects, there is a need to ensure that the chosen process model is based on sound foundation and has a potential to improve the core functions of the industry.

VDC involves much more than simply implementing new software; it is a new way of working (Mandujano et al. 2015). This requires a move away from traditional workflow, with all parties sharing and effectively working on a common pool of information (Mandujano et al. 2016). Lean implementation involves three components: product, organization and process (Kunz and Fischer 2011). The philosophy and culture of Lean and VDC principles have great synergies and share many main ideas (Alarcon et al. 2013). VDC eliminates waste but also improves workflow for many actors, even those who do not use VDC directly (Eastman et al. 2018). VDC encourages and provides a path for the sharing of information among the stakeholders. Although each approach can be carried out independently, to reach a higher potential, it is necessary to consider the culture, philosophy, and technology jointly. This makes the potential for VDC and Lean implementation greater than the sum of their parts, consequently improving project performance (Alarcon et al. 2013).

\section{METHOD}

The relevant articles published during the period from 2000 to 2020 were identified through a systematic search of many electronic databases. In order to limit this broad scope, we performed a keyword search in top journals as well databases and conference proceedings. The search was conducted using three keywords: BIM, VDC, and Lean. These keywords were chosen because we aimed to identify essential components of current literature reviews between VDC and Lean. The studies were divided according to their various methodologies: surveys/interviews, case studies, literature reviews, and implementation guides. The literature on VDC implementation covered many important aspects, including - but not limited to - its benefits and obstacles, synergies between Lean and VDC, its current status, implementation strategies, and the impacts of VDC in the AEC industry (Alarcon et al. 2013). A total of 300 articles that were reviewed in English and contained the selected keywords in their text or abstracts were retrieved through the database searches. Then, the abstracts of the retrieved articles were reviewed to determine whether they met these review's inclusion criteria. The inclusion criteria included systematic integrated literature reviews that (a) used and described systematic search methods, (b) were relevant to VDC and Lean practices, and (c) included new interactions between VDC and Lean. Through this process, 250 articles were selected based on their abstracts. Then, the full text of all 250 articles was reviewed to determine whether they met the inclusion criteria. Finally, a total of 196 articles were selected and included in this literature review. The majority of these articles are from the Center for Integrated Facility Engineering (CIFE), Automation in Construction, and the Journal of Construction Engineering and Management. A substantial difference in the number of published articles can be observed between 2000 and 2020 (Figure 1). The authors found few articles published between 2000 and 2005. The greatest number of articles was published between the years 2020-2016. 


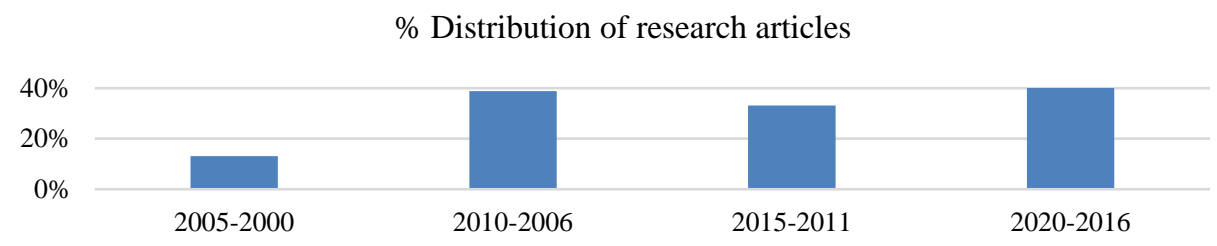

Figure 1: Distribution of research articles.

\section{RESULTS}

Using the information mentioned above, a matrix was developed (Table 1) showing 405 interactions between VDC and Lean, some of which, in one way or another, have been mentioned by Sacks et al. (2010) (including those referred to as 'not found yet' - the full list of interactions can be accessed at: www.maroconsulting.mx). The numbers within tables 1-2 represent types of interaction and if the same type of interaction was found in two papers it was counted once). We focused mostly on those VDC features most mentioned in our literature review to create the tables and matrix: Table 1 shows the frequency of occurrence of each interaction. The Lean principles were chosen based on Koskela (1992) and Sacks et al. (2010). The types of waste that VDC-Lean interaction could reduce and also the Lean techniques that the industry could apply in order to improve VDC adoption (Koskela 1992) were considered. The columns show the total occurrence for each VDC feature, and the rows show the totals for the lean principles. We can see that online communication product/ process $(\mathrm{M})$ is the VDC feature most mentioned in our literature review. Followed by construction planning/ 4D modeling. Also, reduce time (d) and transparency (h) are the most mentioned lean principles allowed by VDC. The interactions more mentioned were: visualization of the design - reduce time, and online communication production/ process - reduce time. It is important to understand the relationship between Lean and VDC.

Dave et al. (2013) have presented four mechanisms to analyze how Lean and BIM relate to each other. In order to test these mechanisms, they were associated with the evidence from practice and/or research presented in the literature. We proposed that VDC allows for more interactions with Lean:

a) VDC contributes directly to Lean goals.

b) VDC enables Lean processes and contributes indirectly to Lean goals.

c) Auxiliary information systems, enabled by VDC, contribute directly and indirectly to Lean goals.

d) Lean processes facilitate the introduction of VDC.

Table 2 shows the frequency of the mechanisms vs. the frequency found in the literature carried on in our methodology about VDC. The hypothesis that, "BIM contributes directly to Lean goals." had the highest frequency within the analysis, followed by mechanisms 2,3 , and 4 . In the fourth column, the hypothesis that, "VDC contributes directly to Lean goals" occurred with the highest frequency, followed by mechanisms b, c, and d. The results suggest that to achieve more synergies between Lean Construction and VDC, including BIM (product), process and organizational modeling, it is necessary to use the entire VDC framework. This allows more positive interactions between lean and VDC, versus a similar situation that only includes an interaction between Lean Construction and BIM. 
Table 1: Frequency of interactions

\begin{tabular}{|c|c|c|c|c|c|c|c|c|c|c|c|c|c|c|c|c|c|c|c|c|}
\hline \multirow[b]{3}{*}{ ID } & \multirow[t]{3}{*}{ LEAN PRINCIPLES } & \multirow{3}{*}{\multicolumn{3}{|c|}{ POP }} & \multicolumn{12}{|c|}{ PRINCIPLES FOR FLOW PROCESS DESIGN AND IMPROVEMENT } & \multicolumn{2}{|c|}{$\begin{array}{l}\text { PROBLEM } \\
\text { SOLVING }\end{array}$} & \multirow[b]{2}{*}{\begin{tabular}{|c|} 
DEVELOPING \\
PARTNERS \\
z \\
\end{tabular}} & \\
\hline & & & & & 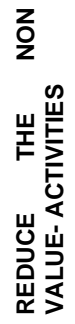 & 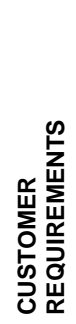 & 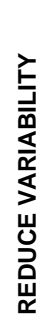 & 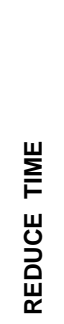 & 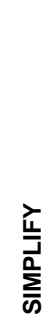 & 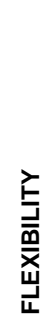 & 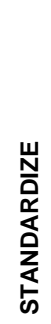 & 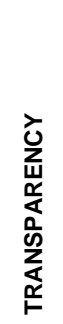 & 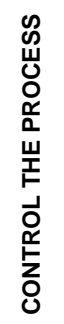 & 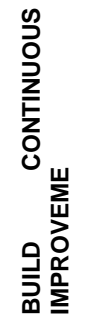 & 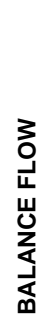 & 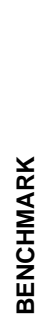 & 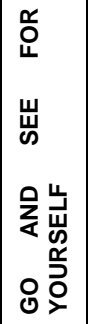 & 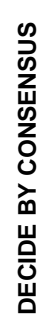 & & \\
\hline & & & & & $\mathbf{a}$ & b & c & d & e & $f$ & g & h & $\mathbf{i}$ & j & $\mathbf{k}$ & I & $\mathrm{m}$ & $\mathrm{n}$ & $\circ$ & Total \\
\hline A & $\begin{array}{l}\text { VISUALIZATION OF THE } \\
\text { DESIGN }\end{array}$ & $\mathrm{X}$ & & & $11^{*}$ & 10 & 20 & 32 & 8 & 6 & 4 & 25 & 9 & 13 & 6 & 1 & 4 & 7 & 6 & 162 \\
\hline B & $\begin{array}{ll}\text { PRODUCTION } & \text { OF } \\
\text { CONSTRUCTION } & \\
\text { DOCUMENTS } & \end{array}$ & $\mathrm{x}$ & & & $15^{\star}$ & 9 & 19 & $24^{*}$ & $9^{*}$ & 6 & 9 & 12 & 9 & 8 & 5 & 2 & 3 & 4 & 10 & 144 \\
\hline C & $\begin{array}{l}\text { ANALYSIS OF DESIGN } \\
\text { OPTIONS }\end{array}$ & $\mathrm{X}$ & & & 8 & 9 & 5 & 9 & 4 & $3^{*}$ & $4^{*}$ & 7 & 3 & 3 & 2 & 1 & 2 & 7 & 4 & 71 \\
\hline D & $\begin{array}{ll}\text { SUPPLY } & \text { CHAIN } \\
\text { MANAGEMENT }\end{array}$ & & $\mathrm{x}$ & & 4 & 2 & 3 & 7 & 3 & 2 & 2 & 2 & 3 & 1 & 2 & 1 & & & 2 & 34 \\
\hline E & DESIGN CHECKING & $\mathrm{X}$ & & & 7 & 4 & 9 & 9 & 4 & 3 & 2 & 9 & 4 & 3 & 2 & 1 & 1 & 1 & 2 & 61 \\
\hline $\mathbf{F}$ & CODE REVIEWS & & $\mathrm{X}$ & & & 1 & & 3 & 1 & & 1 & 3 & 1 & 1 & & 1 & 1 & & 1 & 14 \\
\hline $\mathbf{G}$ & FORENSIC ANALYSIS & & $\mathrm{x}$ & & & 1 & & 2 & 1 & 1 & 1 & 4 & & & & & & & & 10 \\
\hline H & FACILITIES MANAGEMENT & & $\mathrm{x}$ & & 2 & 1 & 2 & 3 & 3 & 1 & 2 & 6 & 3 & 2 & 1 & 1 & 1 & 1 & 1 & 30 \\
\hline $\mathrm{I}$ & $\begin{array}{l}\text { QUANTITY TAKEOFF AND } \\
\text { COST ESTIMATING/ 5D } \\
\text { MODELING }\end{array}$ & & $\mathrm{X}$ & & 4 & 5 & 6 & 14 & 3 & 2 & 2 & 4 & 2 & 2 & 1 & 1 & 1 & 1 & 1 & 49 \\
\hline$J$ & $\begin{array}{l}\text { CONSTRUCTION } \\
\text { PLANNING / 4D MODELING }\end{array}$ & & $\mathrm{X}$ & & 16 & 7 & 13 & 27 & 12 & 10 & 10 & 23 & 10 & 7 & 8 & 3 & 3 & 8 & 9 & 166 \\
\hline K & $\begin{array}{l}\text { ORGANIZATIONAL } \\
\text { MODELING }\end{array}$ & & & $\mathrm{x}$ & 3 & & 3 & 5 & 1 & 1 & 2 & 3 & 2 & 2 & 1 & 2 & 1 & 3 & 4 & 33 \\
\hline L & $\begin{array}{l}\text { BUILDING PERFORMANCE } \\
\text { ANALYSIS }\end{array}$ & & $\mathrm{x}$ & $\mathrm{X}$ & 5 & 2 & 2 & 7 & 3 & 3 & 3 & 9 & 3 & 4 & 2 & 4 & 2 & 2 & 2 & 53 \\
\hline $\mathbf{M}$ & $\begin{array}{l}\text { ONLINE COMMUNICATION } \\
\text { PRODUCT/PROCESS } \\
\end{array}$ & & & $\mathrm{X}$ & 8 & 5 & 12 & 32 & 9 & 6 & 5 & 22 & 6 & 11 & 6 & 2 & 5 & 14 & 24 & 167 \\
\hline Total & & & & & 83 & 56 & 94 & 174 & 61 & 44 & 47 & 129 & 55 & 57 & 36 & 20 & 24 & 48 & 66 & \\
\hline
\end{tabular}

Note: Considering space limitations to access to the full list, please visit: www.maroconsulting.mx. Numbers with * symbol represent negative interactions. 
Table 2: VDC features vs. Lean principles

\begin{tabular}{|c|c|c|c|}
\hline $\begin{array}{l}\text { Hypothesis } \\
\text { number }\end{array}$ & Hypothesis description & BIM & VDC \\
\hline $1, a$ & $\begin{array}{l}\text {... contributes directly to } \\
\text { Lean goals. }\end{array}$ & 328 & 686 \\
\hline $2, b$ & $\begin{array}{c}\text {... enables Lean } \\
\text { processes, which } \\
\text { contributes indirectly to } \\
\text { Lean goals. }\end{array}$ & 310 & 653 \\
\hline $3, c$ & $\begin{array}{l}\text { Auxiliary information } \\
\text { systems, enabled by..., } \\
\text { contribute directly and } \\
\text { indirectly to Lean goals. }\end{array}$ & 306 & 580 \\
\hline $4, d$ & $\begin{array}{l}\text { Lean processes facilitate } \\
\text { the adoption and use of... }\end{array}$ & 253 & 468 \\
\hline $1, a$ & $\begin{array}{l}\text {... contributes directly to } \\
\text { Lean goals. }\end{array}$ & 328 & 686 \\
\hline
\end{tabular}

\section{DISCUSSION}

This research distinguished between VDC and BIM. This step was crucial because in order to begin, it was necessary to clarify this ambiguity. Although significant advances have been made with regard to the synergies between BIM and Lean, there was a gap in extending these interactions throughout the VDC methodology (including BIM (product), process and organization modeling). After clarifying both concepts, 405 interactions between VDC and Lean were identified. These allow for the development of new VDC implementation strategies and also provide a broader picture that allows for the construction industry to implement more holistic and substantial improvements in every project phase. The new interactions found in the literature can help to complete the matrix proposed by Sacks et al. (2010) and create new implementation paths.

Some of the evidence found in this research includes:

- Co-locating the design and detailing teams such that detailers worked side-by-side, allowed them to construct designs virtually and resolve conflicts and issues immediately, further facilitating highly integrated project Delivery (e.g., Big Room).

- Extended networks that increase collaboration among firms are more effective at implementing models across organizations.

- 4D improves efficiency and safety. It can help identify bottlenecks, improve flow, and verify and validate process information.

- 5D models, which connect 3D models to a database for quantity take-off, support location-based planning and scheduling. These types of models make it easier to visualize quantities and integrate them into schedules and cash flows.

- With hyperlinks to drawings and documents, the way of obtaining information is standardized. The variability is reduced when you have direct links to the documents you need. 
- As trust within any multi-organizational value network is considered crucial to collaboration, it is argued that companies trusting each other are more likely to share information in order to identify and manage inefficiencies and reduce costs.

- Multi-skilled resources: flexibility, process integration Optimization modeling algorithm - SIMAN code Off-site construction plant.

Our research showed that without the VDC framework, these interactions would be achieved to a lesser extent. This finding was supported by the previously discussed mechanisms (Table 2). The interactions most mentioned in the literature and practice were:

- Co-locating Visualization of the Design- Reduce Time (Ad);

- Online Communication Product/Process- Reduce Time (Md);

- Construction Planning/4D Modeling - Reduce Time (Jd);

- Visualization of the Design- Transparency (Ah);

- Online Communication Product/Process-Cultivate an Extended Network (Mo)

- Construction Planning/4D Modeling - Transparency (Jh);

- Production of Construction Documents - Reduce Time (Bd);

- Online Communication Product/Process- Transparency (Mh)

In fact, we can mention that the interactions listed above have a strong and direct impact between them. First, with the use of VDC the process as a whole becomes more efficient. The "Production of Construction Documents" becomes automatic, this allows to "Reduce Time" when documents are delivered (EEE). The use of "Construction Planning/4D Modeling" and "Cost Estimation/5D Modeling" help to reduce time and add value to projects. Moreover, the use of "Construction Planning/4D Modeling" improves the "Transparency" and "Reduce Time" in the project. Since the "4D Modeling" enables the visualization of the sequence of the project all issues are identify prior construction. This results in cost and time saving on site because of effective planning. VDC is a methodology based on technology, a clear example of this is the interactions "Online Communication Product/Process" - "Cultivate an Extended Network" and "Online Communication Product/Process" - "Reduce Time" and - "Transparency". The use of tools, such as iRoom onsite, plasma screen monitors, iPADs and or Tablet PC's loaded with the latest VDC model, allows for coordination and communication between all stakeholders. This level of visualization is high because it is close to the actual and most updated model version and is available to different levels of the hierarchy especially for onsite workers.

We cannot neglect the negative interactions found "where the use of VDC inhibits implementation of a lean principle (Sacks et al. 2010)".

- Production of Construction Documents - Reduce Non-Value Activities (Ba);

- Production of Construction Documents - Reduce Time (Bd);

- Production of Construction Documents - Simplicity (Be);

- Analysis of Design Options - Flexibility (Cf); and

- Analysis of Design Options - Standardize $(\mathrm{Cg})$.

The negative interactions can be interpreted in several ways. While VDC allows a range of benefits throughout the entire project, the negative interactions are the result of keep seeing VDC just as a technology putting aside the collaborative view (processes and 
persons). An example of this is the interaction "Production of Construction Documents" and "Reduce Non-Value Adding Activities". In many cases, the models that are sent from one entity to another contain many inconsistencies. Such inconsistencies create extra work during the production documents.

One key result is the interaction between "Production of Construction Documents" and "Reduce Time". As mentioned before this interaction has a strong and direct impact, but when there is an abuse of the ease with which drawings can be generated can lead to more versions of drawings and as a consequence an increase time of processing. This result encourages caution when producing construction documents or analyzing design options. The ease with which "Production of Construction Documents" can be detailed creates a negative interaction with "Simplicity". Too much detail in the construction documents increases complexity rather than simplicity. Finally, the interaction of "Analysis of Design Options" and "Flexibility" and "Standardize" are a clear example of the need to incorporate Lean throughout all VDC practice. Mandujano et al. (2016) found several types of waste within current VDC practice and suggested that if teams use Lean methods and focus on elimination of these types of waste (i.e., non-value added processing, motion (excess), inventory (excess), waiting and overproduction), teams can improve VDC practices dramatically and also suggests the use of protocols for sharing models, BIM libraries, meeting protocols or quality protocols in order to remove waste within VDC practice and, in our case, enhance or reverse the negative interactions.

\section{CONCLUSION}

This study contributes to provide a better understanding of the impact of simultaneous implementation of Lean Construction principles along with the VDC approach on various stages of construction projects. Identifying the interrelationship of lean principles with uses and actions performed through VDC provides a broader picture that allows the AEC industry to take a more holistic approach, which can help to obtain substantial improvements in every project phase, by increasing the effectiveness of the methods through a better alignment with relevant lean principles. The distinction between BIM and VDC definitions is also an important step in developing a better understanding of the methods and their associated management principles. By making this distinction clear, a significant number of new interactions between Lean Principles and VDC were found in the literature that can help to complete previous studies available in the literature and create new implementation paths in the future. Our research showed that without the VDC framework, these interactions would be achieved to a lesser extent. In order to support this, we tested the interaction mechanisms, previously mentioned in the literature. Future research should direct attention toward understanding the nature of these interactions in further detail and increasing the frequency of interactions between VDC and Lean. As previously mentioned, although VDC or Lean Principles initiatives can be carried out independently in order to reach a higher potential of these improvement efforts, it is necessary to consider the important synergies that their interactions offer. Only in this way companies and projects can take full advantage of all the benefits that VDC and Lean offer. Much remains to be done in the area of VDC and Lean, the AEC industry is constantly changing, and needs are becoming greater.

\section{REFERENCES}

Alarcon, L. F., Mandujano, M., and Mourgues, C. (2013). "Analysis of the implementation of VDC from a lean perspective: Literature review." Proc., Proc. 21st 
Ann. Conf. of the Int'l. Group for Lean Construction, Int'1. Group for Lean Construction, Fortaleza, Brasil, 781-790.

Dave, B., Koskela, L., Kiviniemi, A., Tzortzopoulos, P., and Owen, R. (2013). "Implementing Lean in Construction: Lean Construction and BIM." CIRIA, London, UK.

Eastman, C., Teicholz, P., Sacks, R., and Liston, K. (2018). BIM handbook: A guide to building information modeling for owners, managers, designers, engineers and contractors, John Wiley \& Sons.

Koskela, L. (1992). Application of the new production philosophy to construction, Stanford university Stanford, CA.

Kunz, J., and Fischer, M. (2011). "Virtual design and construction: themes, case studies and implementation suggestions." CIFE Working Paper \#097, Center for Integrated Facility Engineering, Stanford University, USA.

Kunz, J., and Fischer, M. (2020). "Virtual design and construction." Construction Management and Economics, 38(4), 355-363.

Lee, M., Cheah, W., Lau, S., Lee, X., Abdullahi, A., and Wong, S. "Evaluation of practicality of virtual design and construction (VDC) with 5D building information modelling (BIM) through a case study." Proc., IOP Conference Series: Materials Science and Engineering, IOP Publishing, 012058.

Mandujano, M. (2017). "A Method To Identify Virtual Design And Construction Implementation Strategies From A Lean Construction Perspective." Degree of Doctor in Engineering Science., Pontificia Universidad Catolica De Chile Chile.

Mandujano, M. (2019). "Integration of Historic Building Information Modeling and Valuation Approaches for Managing Cultural Heritage Sites." Proc. 27th Annual Conference of the International Group for Lean Construction (IGLC)Dublin, Ireland, 1433-1444.

Mandujano, M., Alarcón, L., Kunz, J., and Mourgues, C. "Use of virtual design and construction, and its inefficiencies, from a lean thinking perspective." Proc., Proceedings of the 23rd Annual Conference of the International Group for Lean Construction, Perth, Australia.

Mandujano, M., Alarcón, L., Kunz, J., and Mourgues, C. (2016). "Identifying waste in virtual design and construction practice from a Lean Thinking perspective: A metaanalysis of the literature." Revista de la Construcción, 15(3), 107-118.

Sacks, R., Koskela, L., Dave, B. A., and Owen, R. (2010). "Interaction of lean and building information modeling in construction." Journal of construction engineering and management, 136(9), 968-980. 